\title{
Strain Effectiveness of Gate-all-around Silicon Nanowire n-MOSFET Transistors with Physical Analysis
}

\section{Amit Agarwal}

Sikkim Manipal University

Prashanta Chandra Pradhan

Sikkim Manipal University

Bibhu Prasad Swain ( $\sim$ bibhuprasad.swain@gmail.com )

National Institute of Technology Manipur https://orcid.org/0000-0002-6190-7453

\section{Research Article}

Keywords: GAA MOSFET, Core-cell SiGe/SiNWs, Subthresshold swing

Posted Date: July 20th, 2021

DOl: https://doi.org/10.21203/rs.3.rs-711698/v1

License: (c) (1) This work is licensed under a Creative Commons Attribution 4.0 International License.

Read Full License 


\title{
Strain Effectiveness of Gate-all-around Silicon Nanowire n-MOSFET Transistors with Physical Analysis
}

\author{
${ }^{1}$ Amit Agarwal, ${ }^{1}$ Prashanta Chandra Pradhan, ${ }^{2}$ Bibhu Prasad Swain
}

Electronics and Communication Engineering, Sikkim Manipal Institute of Technology,

${ }^{1}$ Sikkim Manipal University, East Sikkim, Rangpo, India

${ }^{2}$ Department of Physics, National Institute of Technology, Langol, Imphal, Manipur, India

Corresponding author email: bibhuprasad.swain@gmail.com, bpswain@nitmanipur.ac.in

$$
\text { \# +91-8016574105 }
$$

\begin{abstract}
In this paper, we have discussed the effects of uniaxial tensile strain on the performance of gate-all-around (GAA) n-MOSFETs over the core cell SiGe structure are investigated for nanowire (NW) channel down to $5 \mathrm{~nm}$ to $10 \mathrm{~nm}$. The strain has shown an increase in current drain due to a decrease in the energy bandgap in the SiNWs which stretched beyond their normal interatomic distance. The surface potential gate to source voltage ( -0.5 to $0.5 \mathrm{~V})$, drain voltage ( 0 to $2 \mathrm{~V}$ ), donor concentration $\left(10^{7} \mathrm{~cm}^{-3}\right.$ to $\left.10^{14} \mathrm{~cm}^{-3}\right)$, acceptor concentration $\left(10^{17} \mathrm{~cm}^{-3}\right.$ to $\left.10^{22} \mathrm{~cm}^{-3}\right)$, and operating temperature ( 0 to $300 \mathrm{~K}$ ) with varying channel length were investigated. Matlab code is used to employ the GAA strained-Si MOSFETs show excellent subthreshold swing and cutoff behavior, and approximately two times current drive and intrinsic transconductance enhancement compared to similar unstrained $\mathrm{Si}$ devices.
\end{abstract}

Keywords: GAA MOSFET, Core-cell SiGe/SiNWs, Subthresshold swing 


\section{Introduction}

Strained silicon technology has enhanced the further scaling down of MOS technology. Mobility enhancement can be done by applying suitable strain and these strain offers high carrier velocity in the channel, which results in high current output. In strained silicon $[1,2]$ there is stretching of silicon atoms due to which interatomic distance is increased. Strained Silicon is done by putting a layer of silicon on Silicon-Germanium (SiGe) substrate. Silicon Atoms align with the atoms of the silicon-germanium layer and stretching takes place which leads to the formation of strained Silicon. When atoms separation increases atomic forces also decrease thus higher mobility, higher switching speed due to an increase in mobility, and lower energy consumption $[3,4,5]$. Gong et al. investigated compressive strain improves the hole mobility which increasing band bending induced due to alteration reduced effective mass, which leads to higher hole mobility and separation increases between LH (Light Hole) and HH (Heavy Hole), which enhances the hole mobility. Moreover, Naka et al. reported the strain effect on the band structure due to the band shifts in the conduction band and the valence band $[6,7]$. Kim et al. reported improvement of the power delay in the GAA CMOS inverters from 25 to $35 \%$ due to increasing strain development in the PMOS [8]. Zhang et al. investigated the effect of band-to-band tunneling current due to increasing of carrier generation rate from unstrained heterojunction device [9]. Elmessary et al. reported improved Si passivated Ge p-channel GAA MOSFET of 45-nm active pitch starting by using 300-mm buffer SiGe strain relaxed device [10]. The above researcher have not reported effect of strained GAA Si-FET with the diameter $10-15 \mathrm{~nm}$ with channel length 5-10 nm using the MATLAB modeling of following, (i) the surface potential gate to source voltage $(-0.5$ to $0.5 \mathrm{~V})$, (ii) drain voltage 
(0 to $2 \mathrm{~V}$ ), (iii) donor concentration $\left(10^{7} \mathrm{~cm}^{-3}\right.$ to $\left.10^{14} \mathrm{~cm}^{-3}\right)$, (iv) acceptor concentration $\left(10^{17} \mathrm{~cm}^{-3}\right.$ to $\left.10^{22} \mathrm{~cm}^{-3}\right)$, and (iv) operating temperature (0 to $\left.300 \mathrm{~K}\right)$ with varying channel length were investigated. Therefore, it is paramount important to investigate the effect of strained Si GAA MOSFET on doping concentration, operation temperature, the effect of potential difference and, electrochemical protentional.

\section{Analytical Modeling of Strained MOSFET}

Fig. 1 shows lattice strain generated the lattice mismatch between two layers, the siliconon-silicon germanium heterojunction. However, the lattice strain can be modulated by altering the composition of materials. However, the incorporation of lower bandgap alloying affects the on/off current of GAA-MOSFET. Moreover, lowering of bandgap can be operated at the lower operational voltage. The analytical model for the device and implement the strain effect to evaluate the strain effect on the bond is given by [11-12]

$a_{\|}=\frac{a_{1} G_{1} h_{1}+a_{2} G_{2} h_{2}}{G_{1} h_{1}+G_{2} h_{2}}$

Where,

$a_{1}$, and $a_{2}$ are the lattice constant, for the $h_{1}$ and $h_{2}$ thickness respectively for the unstrained layers, for the semiconductors of 1 and 2 , and $G_{i}$ is the shear modulus given by

$G_{i}=2\left(c_{11}^{i}+2 c_{12}^{i}\right)\left(1-D_{i} / 2\right)$

$D^{001}=2 \frac{c_{12}}{c_{11}}$

$D^{110}=\frac{c_{11}+3 c_{12}-2 c_{44}}{c_{11}+c_{12}+2 c_{44}}$

$D^{111}=2 \frac{c_{11}+2 c_{12}-2 c_{44}}{c_{11}+2 c_{12}+4 c_{44}}$

Fig. 2 shows the energy band diagram silicon-silicon germanium heterojunction, using Anderson principle. The energy band diagram of the heterojunction depends on the electron 
affinity $(\chi)$, work function $(\phi)$, and ionization potential $\left(i_{p}\right)$ of the semiconductors. A hydrostatic deformation potential for the valence band of semiconductors $[13,14]$ can be observed as

$a_{v}=\frac{d E_{v, a v}}{d \ln \Omega}$

Where $\Delta E_{v, a v}=a_{v} \frac{\Delta \Omega}{\Omega}$

$\Delta \Omega / \Omega=\operatorname{Tr}(\underset{\epsilon}{\leftrightarrow})=\left(\epsilon_{x x}+\epsilon_{y y}+\epsilon_{z z}\right)$ is the fractional volume change in the unit cell of semiconductor.

$\Delta E_{c}=a_{c} \frac{\Delta \Omega}{\Omega}$

The position of the topmost valence band:

$E_{v}=E_{v, a v}+\frac{\Delta_{0}}{3}$

$E_{c}=E_{v}+E_{g}$

$\Delta E_{v, a v}=E_{v, a v}^{B}-E_{v, a v}^{A}$

$E_{v, a v}=E_{v, a v}^{0}+a_{v} \frac{\Delta \Omega}{\Omega}$

$\Delta E_{v, 2}=\frac{1}{3} \Delta_{0}-\frac{1}{2} \delta E_{001}$

$\Delta E_{v, 1}=-\frac{1}{6} \Delta_{0}+\frac{1}{4} \delta E_{001}+\frac{1}{2}\left[\Delta_{0}^{2}+\Delta_{0} \delta E_{001}+\frac{9}{4}\left(\delta E_{001}\right)^{2}\right]^{1 / 2}$

$\Delta E_{v, 3}=-\frac{1}{6} \Delta_{0}+\frac{1}{4} \delta E_{001}-\frac{1}{2}\left[\Delta_{0}^{2}+\Delta_{0} \delta E_{001}+\frac{9}{4}\left(\delta E_{001}\right)^{2}\right]^{1 / 2}$

In these equations, $\delta E_{001}$ is given by

$\delta E_{001}=2 b\left(\epsilon_{z z}-\epsilon_{x x}\right)$

Where, 


$$
\left[\begin{array}{ccc}
\frac{\Delta_{0}}{3}-\frac{1}{8}\left(\delta E_{001}+3 \delta E_{111}\right) & -\frac{\sqrt{3}}{8}\left(\delta E_{001}-\delta E_{111}\right) & \frac{\sqrt{6}}{8}\left(\delta E_{001}-\delta E_{111}\right) \\
-\frac{\sqrt{3}}{8}\left(\delta E_{001}-\delta E_{111}\right) & \frac{\Delta_{0}}{3}+\frac{1}{8}\left(\delta E_{001}+3 \delta E_{111}\right) & \frac{\sqrt{2}}{8}\left(\delta E_{001}+3 \delta E_{111}\right) \\
\frac{\sqrt{6}}{8}\left(\delta E_{001}-\delta E_{111}\right) & \frac{\sqrt{2}}{8}\left(\delta E_{001}+3 \delta E_{111}\right) & -\frac{2}{3} \Delta_{0}
\end{array}\right]
$$

Where,

$$
\begin{aligned}
& \delta E_{001}=4 b\left(\epsilon_{x x}-\epsilon_{z z}\right) \\
& \delta E_{111}=(4 / \sqrt{3}) d \epsilon_{x y} \\
& \Delta E_{c}^{i}=\left(\Xi_{d} \overleftrightarrow{1}+\Xi_{u}\left\{\hat{a}_{i} \hat{a}_{i}\right\}\right): \overleftrightarrow{\epsilon} \\
& \Delta E_{c, a v}=\left(\Xi_{d}+\frac{1}{3} \Xi_{u}\right) \overleftrightarrow{1}: \overleftrightarrow{\epsilon}
\end{aligned}
$$

The splitting of the bands is given by

$$
\begin{aligned}
& \Delta E_{c}^{001}=\frac{2}{3} \Xi_{u}^{\Delta}\left(\epsilon_{z z}-\epsilon_{x x}\right) \\
& \Delta E_{c}^{100,010}=-\frac{1}{3} \Xi_{u}^{\Delta}\left(\epsilon_{z z}-\epsilon_{x x}\right)
\end{aligned}
$$

Strain along [111] leads to:

$$
\begin{aligned}
& \Delta E_{c}^{111}=2 \Xi_{u}^{L} \epsilon_{x y} \\
& \Delta E_{c}^{\overline{1} 11,1 \overline{1} 1,11 \overline{1}}=-\frac{2}{3} \Xi_{u}^{L} \epsilon_{x y}
\end{aligned}
$$

Strain along [110] leads to:

$$
\begin{aligned}
& \Delta E_{c}^{111,11 \overline{1}}=+\frac{2}{3} \Xi_{u}^{L} \epsilon_{x y} \\
& \Delta E_{c}^{\overline{1} 11,1 \overline{1} 1}=-\frac{2}{3} \Xi_{u}^{L} \epsilon_{x y}
\end{aligned}
$$

For our model strained $\mathrm{Si} / \mathrm{SiGe}$ calculations are as follows:

Using the above-mentioned calculation impact on conduction band is written as $[14,16]$

$$
\left(\Delta E_{c}\right)_{s-S i}=0.57 x
$$

Where $\left(\Delta E_{c}\right)_{s-S i}$ is the decrease in $\chi_{S i}$ due to strain 
$\left(\Delta E_{g}\right)_{s-S i}=0.4 x$

Where $\left(\Delta E_{g}\right)_{s-S i}$ is the change in the bandgap of $\mathrm{Si}$ due to lattice strain between $\mathrm{Si} / S i_{1-x} G e_{x}$ heterojunction, where $x$ is the Ge mole fraction in the $S i_{1-x} G e_{x}$ layer.

The decrease in the bandgap of $S i_{1-x} G e_{x}$ on the $S i$ is given by

$\left(\Delta E_{g}\right)_{\text {SiGe }}=0.467 x$

$\varepsilon_{\text {SiGe }}=11.8+4.2 x$

Where $\varepsilon_{S i G e}$ is the permittivity of $S i_{1-x} G e_{x}$.

$\left(V_{F B, f}\right)_{s-S i}=\left(V_{F B, f}\right)_{S i}+\Delta V_{F B, f}$

Where $\Delta V_{F B, f}=\frac{-\left(\Delta E_{c}\right)_{s-S i}}{q}+\frac{\left(\Delta E_{g}\right)_{s-S i}}{q}-V_{T} \ln \left(\frac{N_{V, S i}}{N_{V, S}-S i}\right)$

$\left(V_{F B, f}\right)_{S i}=\phi_{M}-\phi_{S i}$

The built-in-voltage developed across source and drain junctions due to lattice strain are given below:

$V_{b i, S-S i}=V_{b i, S i}+\left(\Delta V_{b i}\right)_{s-S i}$

Fig. 3 shows the cross-sectional view of the strained GAA MOSFET. Due to the symmetry of the device, the Poisson equation of the strained GAA MOSFET can be written as

$\frac{1}{r} \frac{\partial}{\partial r}\left(r \frac{\partial}{\partial r} \phi(r, z)\right)+\frac{\partial^{2}}{\partial z^{2}} \phi(r, z)=q N_{A} / \varepsilon_{S i} \quad 0 \leq z \leq L, 0 \leq r \leq R$

By considering proper boundary conditions above equation can be simplified as

$\phi_{S}(z)=A e^{k z}+B e^{-k z}-\frac{\beta}{k^{2}}, 0 \leq z \leq L$.

Where

$$
A=\frac{V_{b i, S-S i}\left(1-e^{-k L}\right)+V_{D S}+\beta / k^{2}\left(1-e^{-k L}\right)}{e^{k L}-e^{-k L}}
$$


$B=\frac{V_{b i, S-S i}\left(e^{k L}-1\right)-V_{D S}+\beta / k^{2}\left(e^{k L}-1\right)}{e^{k L}-e^{-k L}}$.

\section{Result \& Discussion}

Fig. 4(a\&b) shows the surface potential of silicon nanowires (SiNWs) varied with donor concentration ' $\mathrm{N}_{\mathrm{d}}$ ' and drain in the $\mathrm{SiNWs}_{\mathrm{S}} \mathrm{Si}_{(1-\mathrm{x})} \mathrm{Ge}_{\mathrm{x}}$ core-shell GAA MOSFET varying with different channel lengths ' $\mathrm{L}$ '. The built-in-potential due to doping concentration are

expressed by the relationship $V_{b i}=\frac{k T}{q} \ln \left(\frac{N_{A} N_{D}}{n_{i}^{2}}\right)$, in which, $\mathrm{V}_{\mathrm{bi}}$ has an impact on surface potential ' $\varphi_{s}$ '. The $\varphi_{s}$ increased with increasing of $\mathrm{N}_{\mathrm{d}}$ concentration of source and drain points of GAA MOSFET. For the $\mathrm{N}_{\mathrm{d}}$ concentration is $10^{17} \mathrm{~cm}^{-3}$, the $\varphi_{s}$ reached at $\sim 0.90$ $\mathrm{V}$, whereas, with for an $\mathrm{N}_{\mathrm{d}}$ concentration of $10^{22} \mathrm{~cm}^{-3}$, the $\varphi_{s}$ reached at $\sim 0.93 \mathrm{~V}$. Increasing of channel length (L) from $10 \mathrm{~nm}$ to $15 \mathrm{~nm}$, the shifting of the $\varphi_{s}$ change of $0.2 \mathrm{~V}$. i.e., from $0.90 \mathrm{~V}$ to $1.1 \mathrm{~V}$. However, the maximum of sub-threshold swing, minimum threshold voltage, and maximum leakage are observed in the same doping level.

Fig. 5 shows the plot between surface potential and channel radius of GAA MOSFET with different channel lengths. As we know that charge carriers move through the channel, so with the change in channel dimension, there will be a direct impact on the movement of the carriers and leads to a change in surface potential and current of the device. We also find from the natural length equation $k^{2}=\frac{2 \cdot \varepsilon_{o x}}{\varepsilon_{t} \cdot R^{2} \cdot \ln \left(1+\frac{t_{O x}}{R}\right)}$, that with change in Channel Radius natural length also changes and which impacts device performance. So, with the increase in the physical dimension (Channel Length and Radius) of the device, there is an increase in the surface potential. From Fig.5, we find that there is a change of $0.04 \mathrm{~V}$ as the channel radius is increased from $5 \mathrm{~nm}$ to $10 \mathrm{~nm}$. When the thin film thickness is reduced, the controllability of the gate over the channel becomes stronger in comparison with the 
influence exerted by the source/drain. From the above Fig. 5, we also find that there is a deviation of $0.12 \mathrm{~V}$ when the channel length is varied from 10 to $15 \mathrm{~nm}$.

Fig.6 shows a plot between surface potential vs. temperature with varying channel lengths from 10 to $15 \mathrm{~nm}$. Similar to the channel radius there is an impact in built-in potential $\left(\mathrm{V}_{\mathrm{bi}}\right)$

with the change in temperature ' $\mathrm{T}$ ' given by the equation $V_{b i}=\frac{k T}{q} \ln \left(\frac{N_{A} N_{D}}{n_{i}^{2}}\right)$. From Fig.6 we find that there is a decrease in surface potential by $0.37 \mathrm{~V}$ with the increase of temperature from 0 to $300 \mathrm{~K}$. Also, we analyze that, with the change of channel length from 10 to $15 \mathrm{~nm}$ the surface potential changes from 0.87 to $1.27 \mathrm{~V}$ at $0 \mathrm{~K}$, but at $300 \mathrm{~K}$ the surface potential changes from 0.84 to $0.9 \mathrm{~V}$. So, there is a much higher change in surface potential at a temperature of $0 \mathrm{~K}$ than that of $300 \mathrm{~K}$.

Fig. 7 and Fig. 8 show the output and transfer characteristics of a cylindrical nanowire of $10 \mathrm{~nm}$ channel length, gate insulator used is $\mathrm{SiO}_{2}$ with a thickness of $2 \mathrm{~nm}$. With the increase of $\mathrm{V}_{\mathrm{ds}}$, drain current increases, and with a higher value of $\mathrm{V}_{\mathrm{gs}}$ drain current also increases. The drain current and the channel charge for $\mathrm{V}_{\mathrm{ds}}=0.2 \mathrm{~V}$ are linearly proportional to $\mathrm{V}_{\mathrm{gs}}$, whereas those for $\mathrm{V}_{\mathrm{ds}}=1.0 \mathrm{~V}$ show exponential behavior.

\section{Conclusion}

In this research, we have analytically modeled strain on the cylindrical GAA-MOSFET and analyzed the impact of strain on the surface potential and transfer characteristics of the devices with varying physical parameters. We have seen that surface potential increases with the increase of drain voltage, gate to source voltage, channel radius, oxide thickness, and doping. We also find that with the increase of strain, mobility, and current increase but threshold voltage decreases. So, device doping can be minimized, and proper device 
parameter settings can be done to reduce short channel effects and improve device performance.

Funding: The authors declare no funding for this work.

Conflicts of interest/Competing interests: The authors have no conflict of interest.

Availability of data and material (data transparency)

Code availability (software application or custom code): The author used MATLAB codes

\section{Authors' contributions:}

Amit Agarwal: Planning, Data requisition, and modeling of GAA FET

Prashanta Chandra Pradhan: Supervisor of Amit Agarwal

Bibhu Prasad Swain: Supervisor of Amit Agarwal

Consent for publication: All authors consent to the publication of the manuscript.

Ethics approval: Not applicable

Consent to participate: Not Applicable

Acknowledgments: Not Applicable

\section{Reference}

[1] Aberg I, Olubuyide OO, Chleirigh CN, Lauer I, Antoniadis DA, Li J, R. Hull, and J.L. Hoyt, (2004). Electron and hole mobility enhancements in sub-10 nm-thick strained silicon directly on insulator fabricated by a bond and etch-back technique, VLSI Symp. Tech. Dig.,: 52-53. DOI: 10.1109/VLSIT.2004.1345388.

[2] Coquand R, Casse M, Barraud S, Cooper D, Maffini-Alvaro V., Samson M.-P., Monfray S., Boeuf F., Ghibaudo G., Faynot O., and Poiroux T., (2013). Strain-induced performance enhancement of tri-gate and omega-gate nanowire FETs scaled down to 10-nm width, IEEE Trans. Electron Device, 60(2):727-732. DOI: 10.1109/TED.2012.2231684 
[3] Balslev I., (1966). Influence of uniaxial stress on the indirect absorption edge in silicon and germanium, Phys. Rev, 143:636-647. DOI:https://doi.org/10.1103/PhysRev.143.636

[4] Hashemi P, Gomez L, Hoyt JL (2009) Gate-all-around n- MOSFETs with uniaxial tensile strain-induced performance enhancement scalable to sub-10-nm nanowire diameter, IEEE Electron Device Lett., 30(4):401-403. DOI: 10.1109/LED.2009.2013877.

[5] Herring C, Vogt E., (1956). Transport and deformation-potential theory for many-valley semiconductors with anisotropic scattering, Phys. Rev.,101:944-961. DOI: https://doi.org/10.1103/PhysRev.101.944

[6] Gong X, Han G, Su S, Cheng R, Guo P, Bai F, Yang Y, Zhou Q, Liu B, Goh K H, Zhang G, Xue C, Cheng B, Yeo YC (2013) Uniaxially strained germanium-tin (GeSn) gate-allaround nanowire PFETs enabled by a novel top-down nanowire formation technology, Symp. VLSI Tech. Dig. : T34-T35. DOI:https://doi.org/10.1103/PhysRev.101.944.

[7] Nakamura H, (2009) Strain effects on the band structure for Si nanowires. 2009 9th IEEE Conference on Nanotechnology (IEEE-NANO), 8, 555-558.

[8] Kim K, Lee JH, Oh S (2020) Strain effect on the performance of multi-stacked gate-allaround CMOS inverters, Semicond. Sci. Technol. 35:125021 https://doi.org/10.1088/13616641/abbc8e.

[9] Zhang Y, Li Z (2020) Analytical drain current model of strained junctionless nanowire tunnel field-effect transistor fabricated on $\mathrm{Si}_{1-\mathrm{x}} \mathrm{Ge}_{\mathrm{x}}$ virtual substrate, 14(8):1195 - 1200, DOI: 10.1049/iet-cds.2019.0515. 
[10] L. Witters, Arimura H.; Sebaai F.; Hikavyy A.; Milenin A. P.; Loo; A R.. Keersgieter De; Eneman G.; Schram T., Wostyn K., Devriendt K., Schulze A., Lieten R., Bilodeau S. Strained Germanium Gate-All-Around pMOS (2017) Device Demonstration Using Selective Wire Release Etch Prior to Replacement Metal Gate Deposition, Strained Germanium Gate-All-Around pMOS Device Demonstration Using Selective Wire Release Etch Prior to Replacement Metal Gate Deposition, IEEE Transactions On Electron Devices, 64(11): 4588, DOI: 10.1109/TED.2017.2756671.

[11] Walle C.G. Van de, Martin RM, (1986). Theoretical calculations of heterojunction discontinuities in the Si/Ge system, Phys. Rev. B, 34:5621-5634. DOI:https://doi.org/10.1103/PhysRevB.34.5621

[12] Chris G. Walle V (1989) Band lineups and deformation potential in the model-solid theory. Phys. Rev. B.39:1871-1883. DOI:https://doi.org/10.1103/PhysRevB.39.1871

[13] Kumar MJ, Venkataraman V, Nawal S, (2007) Impact of strain or Ge content on the threshold voltage of nanoscale strained-Si/SiGe bulk MOSFETs, IEEE Trans. Device Mater. Reliab., 7:181-187. DOI: 10.1109/TDMR.2006.889269

[14] Lim JS, Thompson SE, Fossum JG (2004) Comparison of threshold voltage shifts for uniaxial and biaxial tensile-stressed n- MOSFETs, IEEE Trans. Electron Device, 25(11) :731- 733. DOI: 10.1109/LED.2004.837581.

[15] Kumar M J, Venkataraman V, Nawal S, (2007)Analytical Drain Current Model of Nanoscale Strained-Si/SiGe MOSFETs for Analog Circuit Simulation," 20th International Conference on VLSI Design held jointly with 6th International Conference on Embedded Systems (VLSID'07), Bangalore: 189-194, DOI: 10.1109/VLSID.2007.38. 
[16] Uchida K, Krishnamohan T, Saraswat KC, Nishi Y, (2005) Physical mechanisms of electron mobility enhancement in uniaxial stressed MOSFETs and impact of uniaxial stress engineering in ballistic regime, IEDM Tech. Dig.:129-132. DOI: 10.1109/IEDM.2005.1609286 


\section{Figure captions}

Fig. 1. Schematic of strained silicon [1]

Fig. 2 Effect of strain on silicon band structure [15]

Fig. 3 Schematic view of the strained GAA MOSFET

Fig. 4. Surface potential vs channel length with varying $\mathrm{Na}$ and $\mathrm{N}_{\mathrm{d}}$ doping strained MOSFET.

Fig. 5. Surface potential vs channel radius with varying channel length in strained MOSFET.

Fig. 6. Surface potential vs temperature with varying channel length in strained MOSFET

Fig. 7. Change in drain current with change in applied drain voltage gate to source voltage for strained MOSFET

Fig. 8. Change in drain current with change and within the applied gate to source voltage different drain voltage for strained MOSFET. 

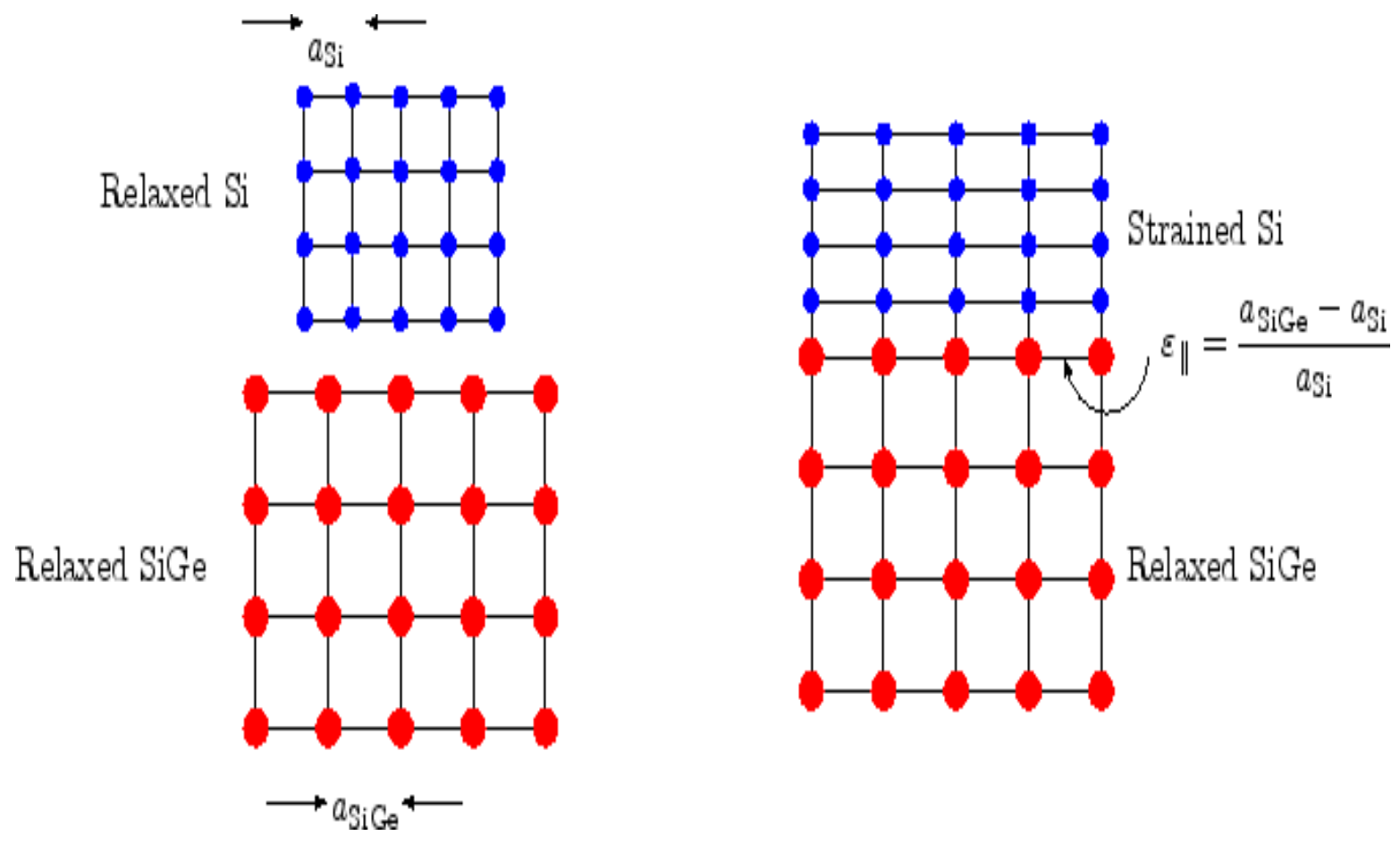

Fig. 1 


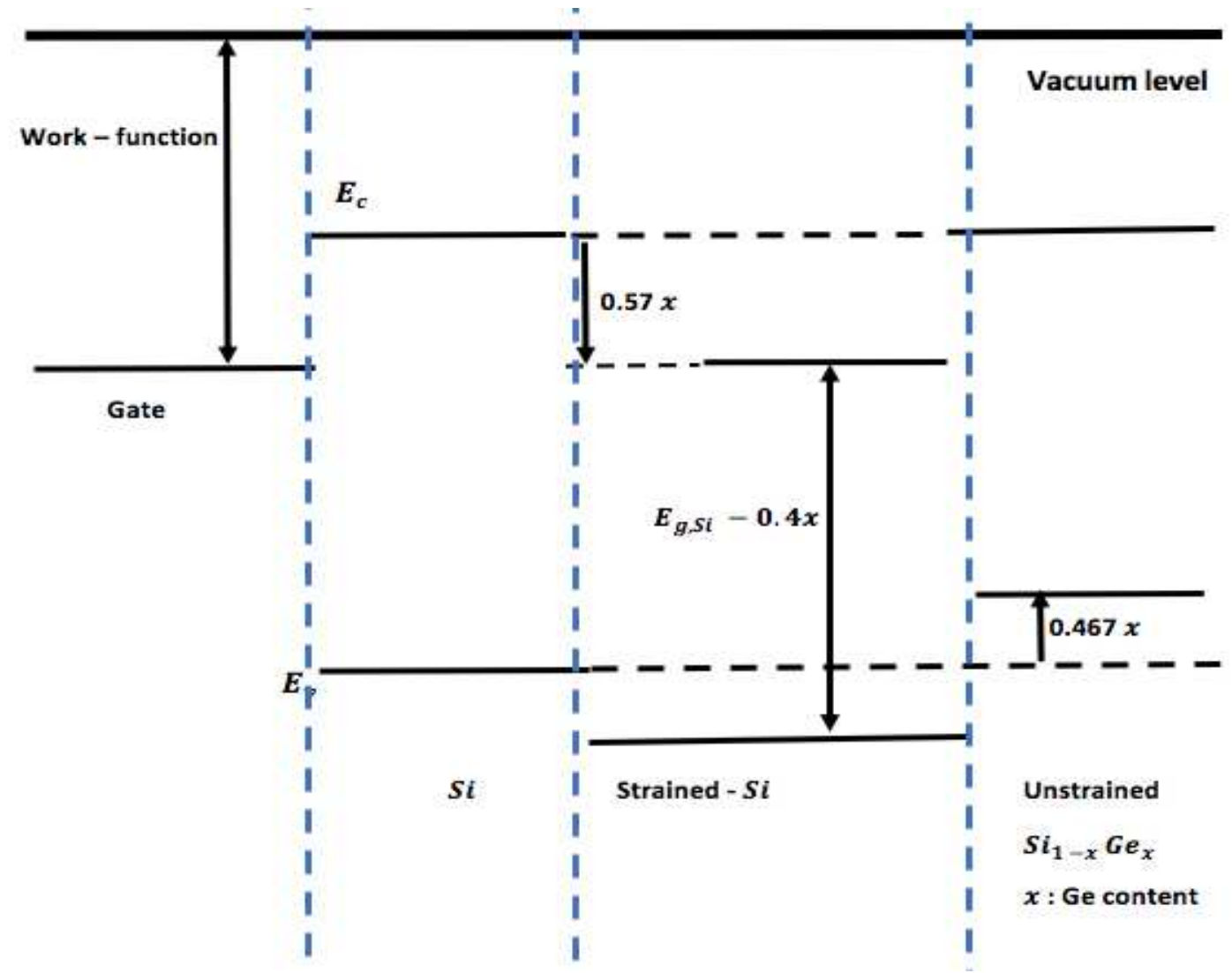

Fig. 2 


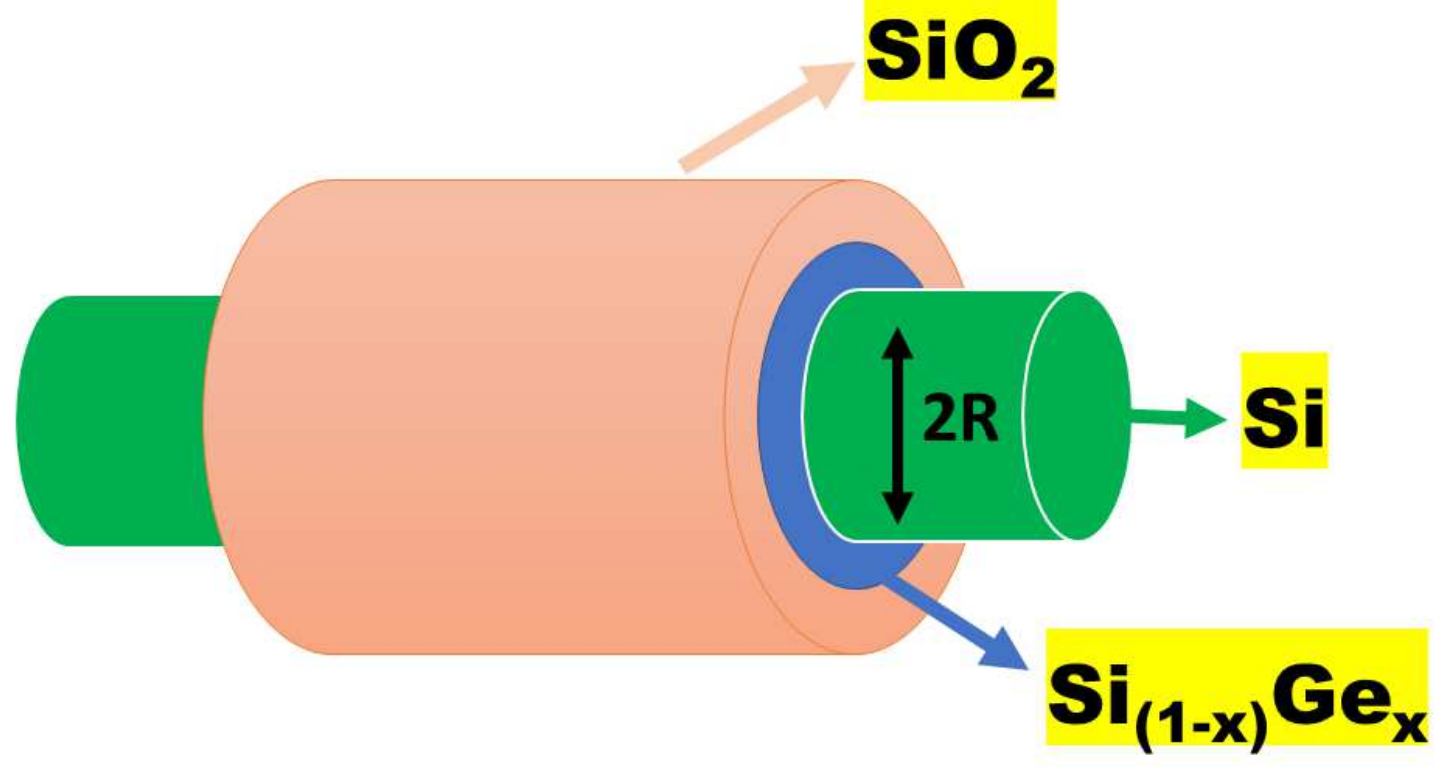

Fig. 3 

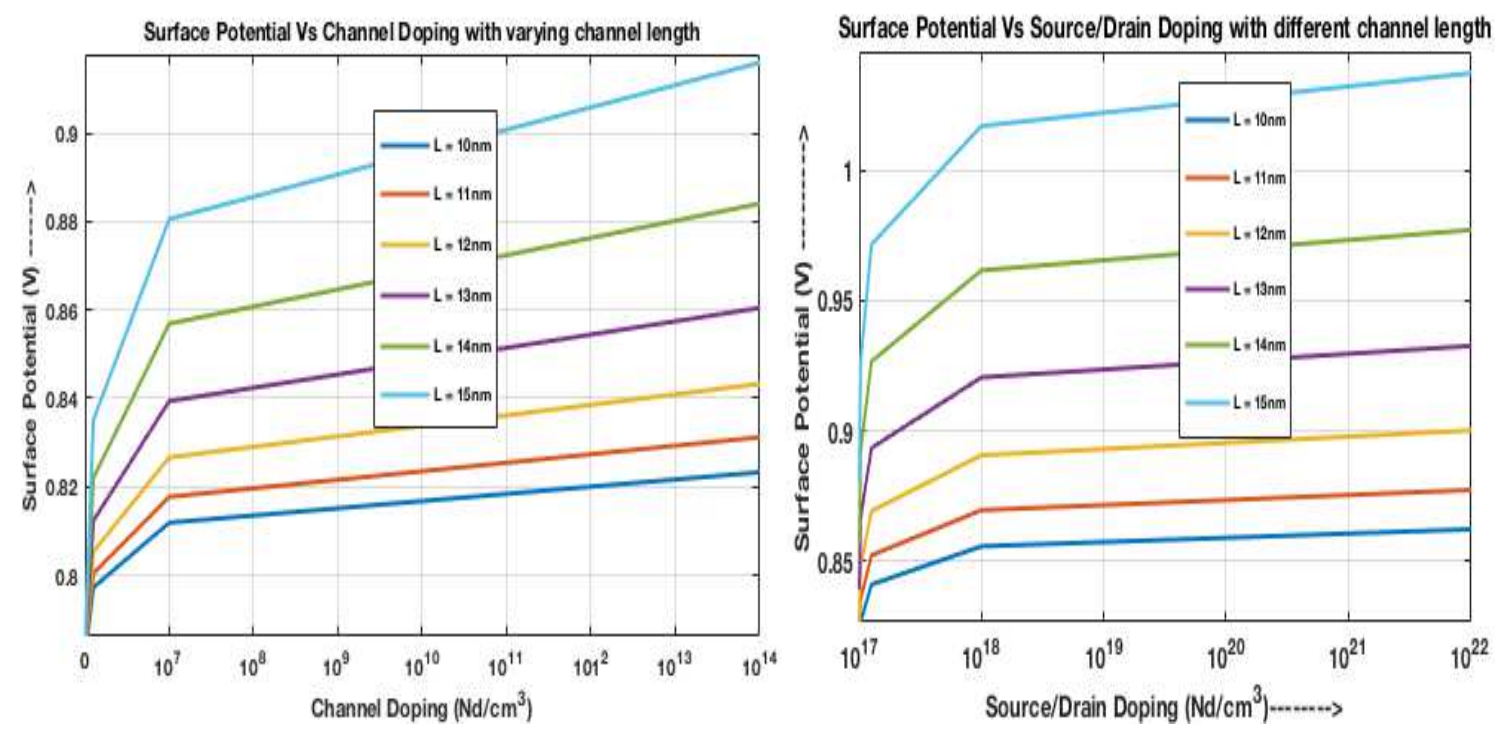

Fig. 4(a\&b) 


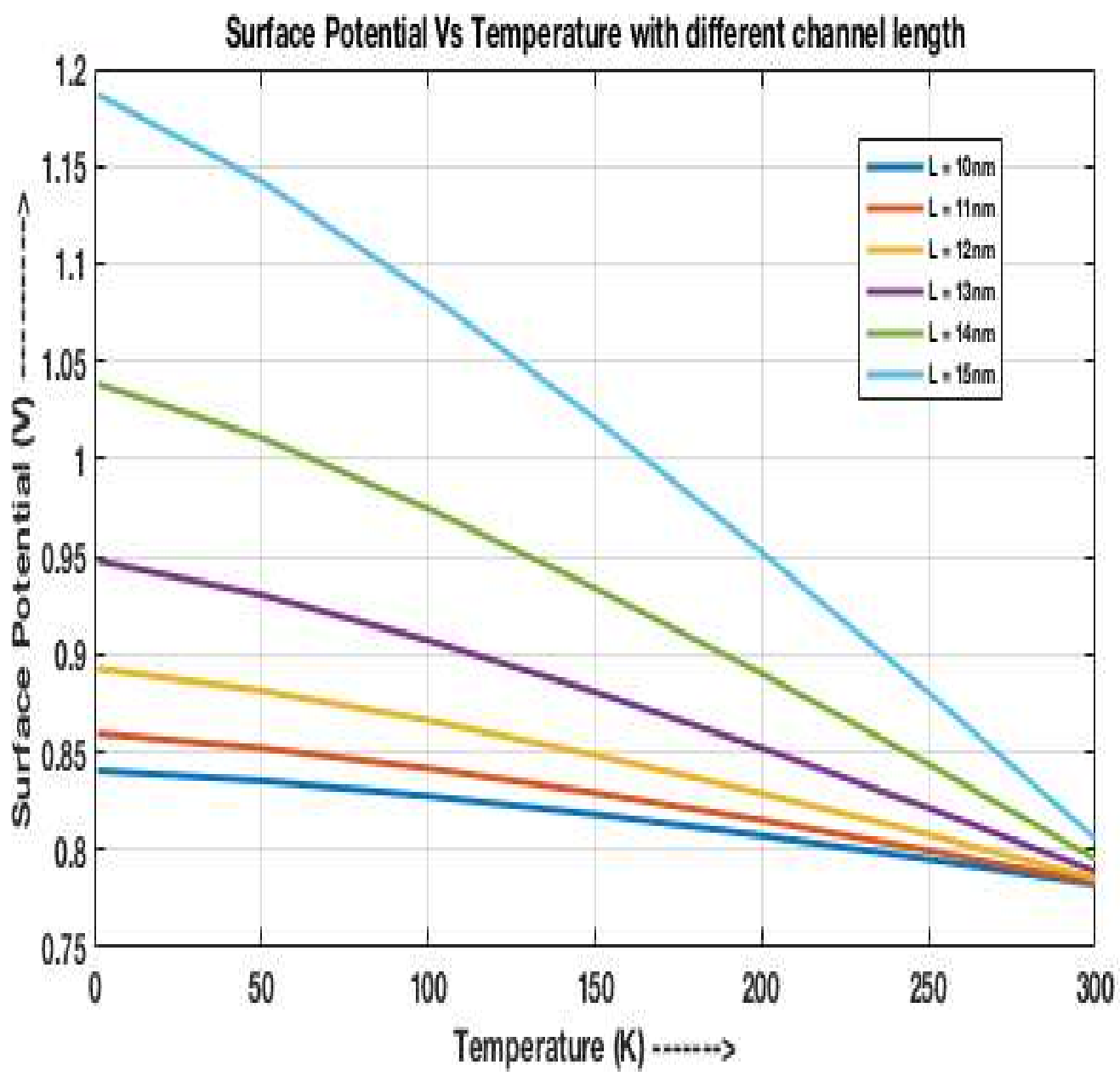

Fig. 5 


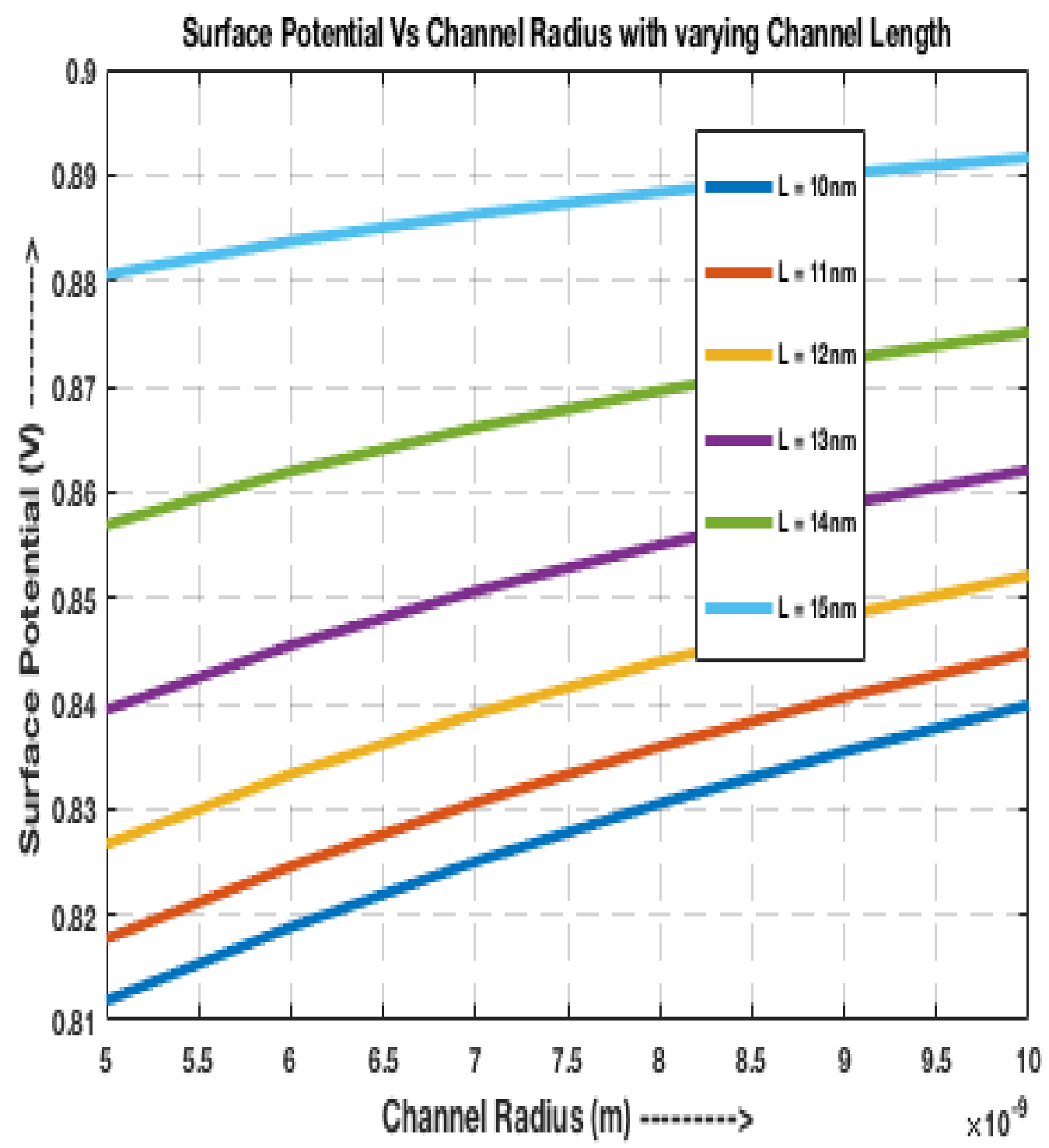

Fig. 6 


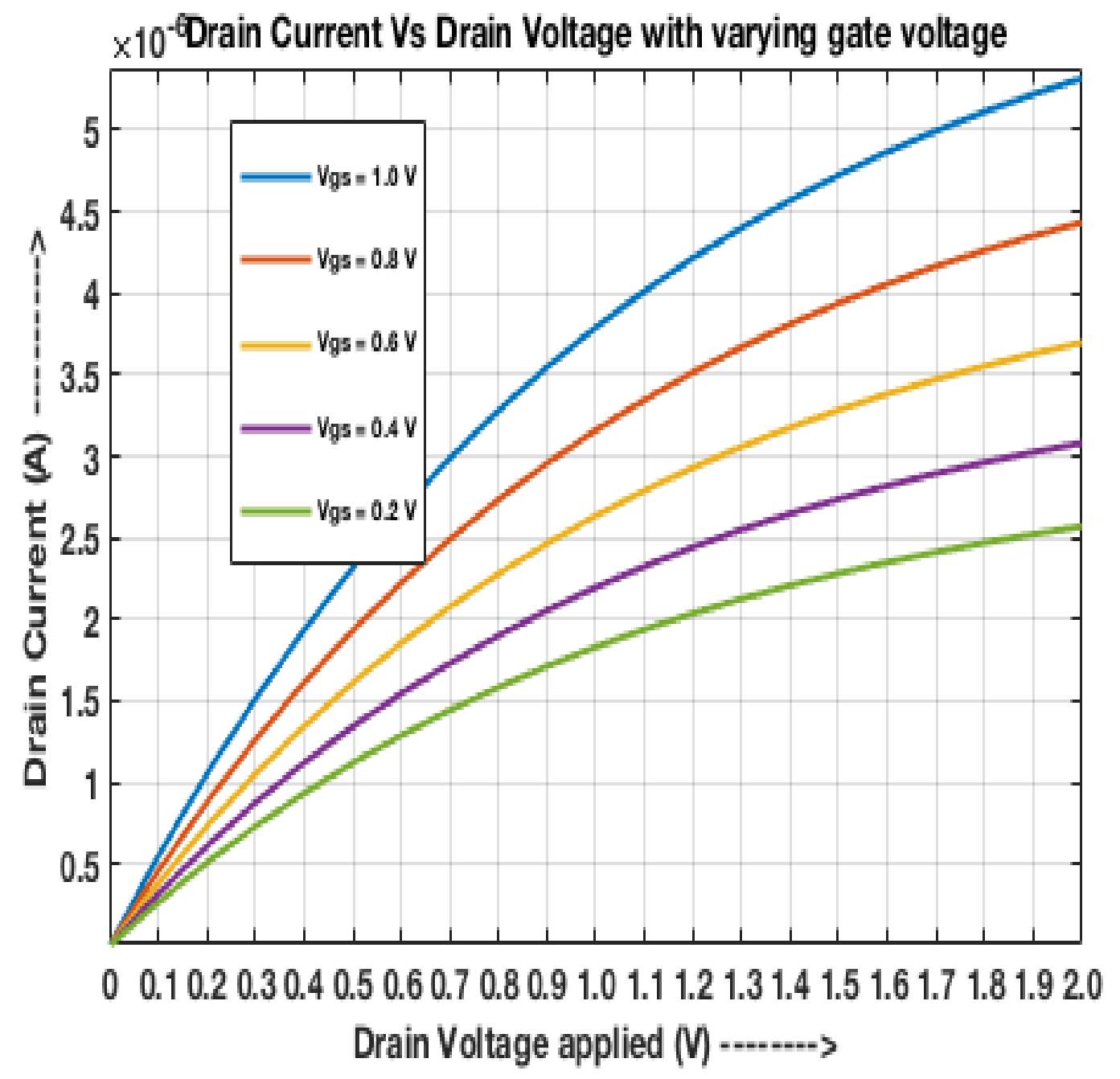

Fig. 7 


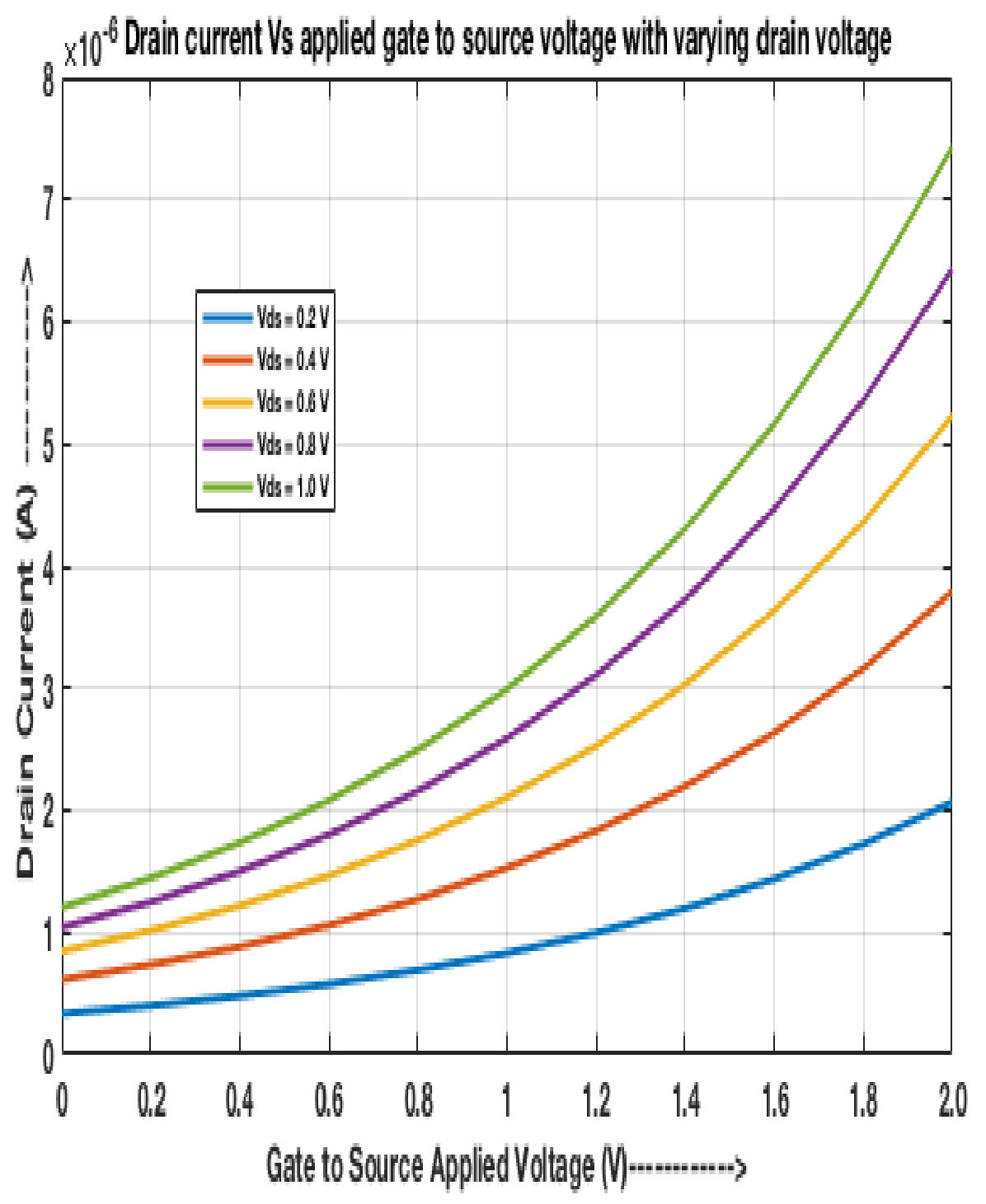

Fig. 8 